\title{
HADAMARD'S INEQUALITY FOR A TRIANGLE, A REgULAR POLYGON AND A CIRCLE
}

\author{
A. MCD. MERCER \\ Abstract. Analogues of Hadamard's integral inequalities are found in two dimensions for the \\ regions stated in the title. \\ Mathematics subject classification (2000): 26D15. \\ Key words and phrases: Hadamard's inequality, convex functions. \\ REFERENCES
}

[1] S. S. Dragomir, On Hadamard's inequality on a disk, J. Ineq. Pure App. Math 1 no. 1 (2000). 